\title{
Inhibiteur de la DPP-4 ou des SGLT2 après échec de la metformine seule dans le diabète de type 2
}

\author{
Prs NICOLAS PAQUOT ${ }^{\mathrm{a}}$ et ANDRÉ J. SCHEEN ${ }^{\mathrm{a}, \mathrm{b}}$
}

Rev Med Suisse 2017; 13: 1410-5

\begin{abstract}
Après échec d'une monothérapie par metformine, les inhibiteurs de la dipeptidyl peptidase-4 (gliptines) et les inhibiteurs des cotransporteurs sodium-glucose de type 2 (gliflozines) offrent une alternative à un traitement par sulfamides, en particulier chez les patients diabétiques à risque d'hypoglycémie. Le choix peut être orienté en fonction des caractéristiques individuelles du patient considéré: plutôt une gliptine chez un patient pas trop obèse ni trop hyperglycémique, relativement âgé, avec un profil fragilisé, éventuellement insuffisant rénal; plutôt une gliflozine chez un patient obèse, hypertendu, hyperuricémique, avec des antécédents cardiovasculaires (insuffisance cardiaque), sans insuffisance rénale avancée et avec un faible risque d'infections urogénitales ou de phénomènes de déshydratation/hypotension.
\end{abstract}

DPP-4 or SGLT2 inhibitor added to metformin alone in type 2 diabetes

After failure of a monotherapy with metformin, dipeptidyl peptidase-4 inhibitors (gliptins) and sodium-glucose cotransporters type 2 (gliflozins) offer an alternative to the add-on of a sulphonylurea, especially in diabetic patients at risk of hypoglycaemia. The choice between a gliptin and a gliflozin may be guided by the individual patient characteristics: rather a gliptin in a patient without obesity or severe hyperglycaemia, in an elderly patient, with a frailty profile or with renal impairment; rather a gliflozin in an obese patient, with hypertension, hyperuricaemia, antecedents of cardiovascular disease (especially heart failure), without advanced renal insufficiency and with a low risk of urinary/genital infections or events linked to dehydration such as hypotension.

\section{INTRODUCTION}

Le traitement du diabète de type 2 (DT2) s'est considérablement complexifié au cours des dernières années et le choix entre de nombreuses classes pharmacologiques est désormais possible, essentiellement en bi ou trithérapie, ${ }^{1-3}$ comme discuté dans un article précédent de la revue. ${ }^{4}$ Pendant longtemps, mis à part l'insuline, le praticien avait le choix entre un biguanide (metformine) et un sulfamide hypoglycémiant, pour aboutir à une combinaison des deux en cas d'échec de la monothérapie initiale. ${ }^{5}$ Depuis la publication de l'étude UKPDS (United Kingdom Prospective Study) en 1998, ${ }^{6}$ la metformine s'est imposée comme premier choix médicamen-

aUniversité de Liège, Service de diabétologie, nutrition et maladies métaboliques, CHU Liège, ${ }^{b}$ Unité de pharmacologie clinique, CHU Sart Tilman, 4000 Liège, Belgique teux chez tout patient DT2, sauf s'il existe une intolérance digestive ou une contre-indication à son utilisation. ${ }^{1-3} \mathrm{La}$ prescription de la metformine en présence d'une insuffisance rénale a cependant été élargie récemment puisqu'il est admis dorénavant que le biguanide peut être prescrit chez des patients DT2 avec un débit de filtration glomérulaire (DFG) entre 30 et $60 \mathrm{ml} / \mathrm{min} / 1,73 \mathrm{~m}^{2}$, à condition de réduire la dose de moitié et de surveiller la fonction rénale régulièrement de façon à interrompre ce traitement si le DFG tombe en dessous de $30 \mathrm{ml} / \mathrm{min} / 1,73 \mathrm{~m}^{2} .^{7}$ Après l'échec d'une monothérapie par metformine, bien entendu en association avec les mesures hygiéno-diététiques, plusieurs choix s'offrent à présent au praticien et les sulfamides sont de plus en plus concurrencés et de moins en moins utilisés en deuxième ligne, avec la recommandation d'une «utilisation prudente» (use with caution). ${ }^{2}$ En effet, si les sulfamides ont assez bien résisté face à la commercialisation des inhibiteurs des alpha-glucosidases (acarbose), des glinides (répaglinide) et des thiazolidinediones (glitazones), ils doivent maintenant faire face à deux nouvelles classes d'antidiabétiques oraux, les inhibiteurs de la dipeptidyl peptidase-4 (DPP-4), mieux connus sous le nom de gliptines, ${ }^{8}$ et les inhibiteurs de cotransporteurs sodiumglucose de type 2 (SGLT2) rénaux, appelés aussi gliflozines. ${ }^{9}$ Ces deux classes pharmacologiques comportent différentes molécules, soit de façon isolée, soit en combinaisons fixes avec la metformine (tableau 1). Elles ont chacune prouvé

\begin{tabular}{l|c|c|l}
\hline & \multirow{2}{*}{ TABLEAU 1} & $\begin{array}{c}\text { Inhibiteurs de la DPP-4 } \\
\text { et des SGLT2 }\end{array}$ & \\
\hline
\end{tabular}

Présentations individuelles, en combinaison libre avec la metformine ou en associations fixes.

\begin{tabular}{|c|c|c|}
\hline $\begin{array}{l}\text { Inhibiteurs de la DPP-4 } \\
\text { (gliptines) }\end{array}$ & Dosages disponibles & $\begin{array}{l}\text { Combinaisons fixes } \\
\text { avec la metformine }\end{array}$ \\
\hline $\begin{array}{l}\text { Sitagliptine (Januvia) } \\
\text { Vildagliptine (Galvus) } \\
\text { Saxagliptine (Onglyza) } \\
\text { Linagliptine (Trajenta) } \\
\text { Alogliptine (Vipidia) }\end{array}$ & $\begin{array}{l}100,50 \text { et } 25 \mathrm{mg} \\
50 \mathrm{mg} \\
5,2,5 \mathrm{mg} \\
5 \mathrm{mg} \\
25,12,5 \text { et } 6,25 \mathrm{mg}\end{array}$ & $\begin{array}{l}\text { Janumet } \\
\text { Eucreas } \\
\text { Komboglyze } \\
\text { Jentadueto } \\
\text { Vipdomet }\end{array}$ \\
\hline $\begin{array}{l}\text { Inhibiteurs des SGLT2 } \\
\text { (gliflozines) }\end{array}$ & Dosages disponibles & $\begin{array}{l}\text { Combinaisons fixes } \\
\text { avec la metformine }\end{array}$ \\
\hline $\begin{array}{l}\text { Canagliflozine (Invokana) } \\
\text { Dapagliflozine (Forxiga) } \\
\text { Empagliflozine (Jardiance) }\end{array}$ & $\begin{array}{l}100,300 \mathrm{mg} \\
10 \mathrm{mg} \\
10,25 \mathrm{mg}\end{array}$ & $\begin{array}{l}\text { Invokamet } \\
\text { Xigduo } \\
\text { Synjardy }\end{array}$ \\
\hline Gliptine + gliflozine & Dosages disponibles & Combinaisons fixes \\
\hline $\begin{array}{l}\text { Saxagliptine + dapagliflo- } \\
\text { zine } \\
\text { Linagliptine + empagli- } \\
\text { flozine }\end{array}$ & $\begin{array}{l}5 \mathrm{mg}+10 \mathrm{mg} \\
5 \mathrm{mg}+25 \mathrm{mg}\end{array}$ & $\begin{array}{l}\text { Qtern } \\
\text { Glyxambi }\end{array}$ \\
\hline
\end{tabular}


leurs efficacité et sécurité en combinaison avec d'autres médications antidiabétiques, dont la metformine. ${ }^{10}$

Le but de cet article est de discuter les avantages et les inconvénients d'ajouter, soit une gliptine, soit une gliflozine, chez un patient dont le DT2 est insuffisamment contrôlé par metformine prescrite à la dose usuelle maximale tolérée, situation fréquemment rencontrée en clinique. ${ }^{11}$ Les critères de choix ont été discutés en faisant référence à un cas clinique précis dans une contribution récente. ${ }^{12}$

\section{ARGUMENTS EN FAVEUR DE LA PRESCRIPTION D'UN INHIBITEUR DE LA DPP-4}

Les inhibiteurs de la DPP-4 offrent plusieurs avantages par rapport aux sulfamides, parmi lesquels les quatre suivants sont le plus souvent mis en exergue. ${ }^{13,14}$ L'avantage le plus évident est l'absence de risque hypoglycémique puisque la sécrétion d'insuline est stimulée de façon gluco-dépendante, contrairement au mécanisme d'action des sulfamides. Le deuxième réside dans la neutralité pondérale, contrastant avec la prise de poids généralement observée avec les sulfamides. ${ }^{15}$ Le troisième concerne une meilleure durabilité de l'effet anti-hyperglycémiant. En effet, si celui des inhibiteurs de la DPP-4 est moins puissant durant les premiers mois, il se maintient de façon plus prolongée alors que les sulfamides s'exposent à un échappement plus ou moins rapide. Enfin, le quatrième fait référence à un moindre risque d'interactions médicamenteuses, source de manifestations indésirables. ${ }^{16}$

Mais il existe également d'autres avantages des gliptines par rapport aux sulfamides, en particulier lors de leur utilisation dans des populations spéciales, dont les patients âgés, ceux avec insuffisance rénale ou encore ceux avec antécédents de maladie cardiovasculaire. Incontestablement, les études menées avec les sulfamides dans ces différentes populations sont insuffisantes et ne permettent pas d'être rassurés quant à leur utilisation avec une sécurité suffisante. Inversement, les gliptines ont été particulièrement bien étudiées et ont apporté des résultats rassurants quant à leur efficacité et à leur sécurité d'emploi. ${ }^{17}$ Ainsi, les gliptines ont été testées chez des patients âgés, caractérisés par une plus grande fragilité, avec des résultats favorables. ${ }^{18}$ Elles ont également démontré leur efficacité et leur sécurité d'emploi chez les patients DT2 avec une insuffisance rénale et ce, à tous les stades. Les posologies de la sitagliptine, de la vildagliptine, de la saxagliptine et de l'alogliptine doivent être diminuées en fonction de la valeur du DFG; seule la linagliptine, qui présente une élimination hépatobiliaire, peut être poursuivie à la même dose. ${ }^{19}$ Comme la metformine peut désormais être utilisée chez les patients DT2 avec une insuffisance rénale de grade 3, il existe donc une opportunité de combiner metformine et gliptine chez ce type de patient.?

Enfin, les inhibiteurs de la DPP-4 ont démontré leur sécurité cardiovasculaire, contrairement aux sulfamides pour lesquels la controverse persiste. ${ }^{20}$ Ainsi, une non-infériorité par rapport à un placebo (prouvant ainsi la sécurité cardiovasculaire des inhibiteurs de la DPP-4, comme demandé par la Food and Drug Administration américaine) a été objectivée dans trois grands essais cliniques prospectifs concernant la saxagliptine,

\begin{tabular}{|c|c|c|c|}
\hline & TABLEAU 2 & \multicolumn{2}{|c|}{$\begin{array}{l}\text { Prescription d'un inhibiteur de la DPP-4 } \\
\text { ou d'un inhibiteur des SGLT2 }\end{array}$} \\
\hline Arguments & \multicolumn{2}{|c|}{ Inhibiteurs de la DPP-4 } & Inhibiteurs des SGLT2 \\
\hline Pour & \multicolumn{2}{|c|}{$\begin{array}{l}\text { - Absence d'hypoglycémie } \\
\text { - Meilleure durabilité } \\
\text { qu'un sulfamide } \\
\text { - Neutralité pondérale } \\
\text { - Excellente tolérance } \\
\text { - Sécurité chez les sujets âgés } \\
\text { - Sécurité chez les sujets } \\
\text { insuffisants rénaux } \\
\text { - Sécurité chez les sujets } \\
\text { avec maladie cardiovasculaire }\end{array}$} & $\begin{array}{l}\text { - Absence d'hypoglycémie } \\
\text { - Bonne puissance anti- } \\
\text { hyperglycémiante } \\
\text { - Perte pondérale } \\
\text { - Diminution de la pression } \\
\text { artérielle } \\
\text { - Diminution de l'uricémie } \\
\text { - Protection cardiovasculaire } \\
\text { - Protection rénale } \\
\text { - Association possible } \\
\text { avec un agoniste des récep- } \\
\text { teurs du GLP-1 }\end{array}$ \\
\hline Contre & \multicolumn{2}{|c|}{$\begin{array}{l}\text { - Un peu moins puissants } \\
\text { (si HbA1c }>8 \% \text { ) } \\
\text { - Pas de perte pondérale } \\
\text { - Pas de protection } \\
\text { cardiovasculaire } \\
\text { - Incertitude sur pancréatite? } \\
\text { - Incertitude sur insuffisance } \\
\text { cardiaque? (saxagliptine) }\end{array}$} & $\begin{array}{l}\text { - Perte d'efficacité } \\
\text { si DFG < } 60 \mathrm{ml} / \mathrm{min} / 1,73 \mathrm{~m}^{2} \\
\text { - Infections uro-génitales } \\
\text { - Risque de déshydratation/hy- } \\
\text { potension } \\
\text { - Prudence chez le sujet âgé } \\
\text { fragile } \\
\text { - Risque d'acidocétose (rare) } \\
\text { - Risque de fractures? } \\
\text { (canagliflozine) } \\
\text { - Risque d’amputations } \\
\text { des orteils? (canagliflozine) }\end{array}$ \\
\hline
\end{tabular}

l'alogliptine et la sitagliptine, respectivement dans SAVORTIMI 53, EXAMINE et TECOS, comme discuté dans un article précédent. ${ }^{21}$ Cependant, aucun de ces essais n'a démontré une supériorité, c'est-à-dire a mis en évidence une réduction des événements cardiovasculaires majeurs par comparaison au groupe traité par placebo, ${ }^{21}$ alors qu'une réduction significative, y compris de la mortalité cardiovasculaire et de la mortalité de toutes causes, a été rapportée avec l'empagliflozine, un inhibiteur sélectif des SGLT2, dans l'étude EMPA-REG OUTCOME. ${ }^{22,23}$

Les arguments en faveur et en défaveur de la prescription d'un inhibiteur de la DPP-4 après échec d'une monothérapie par metformine sont résumés dans le tableau 2.

\section{ARGUMENTS EN FAVEUR DE LA PRESCRIPTION D'UN INHIBITEUR DES SGLT2}

Les inhibiteurs des SGLT2 agissent indépendamment de l'insuline, en abaissant le seuil rénal de la réabsorption du glucose et en forçant une glucosurie. ${ }^{9,24}$ En réduisant la glucotoxicité, ils améliorent, indirectement, la sécrétion insulinique résiduelle et la sensibilité tissulaire à l'insuline (tableau 3$)^{25}$ Comme les inhibiteurs de la DPP-4, ils n'entraînent pas d'hypoglycémie lorsqu'ils sont ajoutés à un traitement par metformine (les deux classes n'augmentent légèrement ce risque que si elles sont ajoutées à un traitement par sulfamide ou par insuline) ${ }^{26}$ Les gliflozines s'avèrent abaisser les taux d'hémoglobine glyquée ( $\mathrm{HbAlc}$ ) de façon un peu plus marquée que les gliptines, surtout si le niveau initial d'HbAic est plus élevé (> 8,5\% ou $69 \mathrm{mmol} / \mathrm{mol}$ ) alors que l'efficacité est assez comparable si le taux d'HbAic n'est que modérément accru (aux environs de 7,5-8\% ou 58-64 $\mathrm{mmol} / \mathrm{mol}$ ). Cette différence s'explique par le fait que l'importance de la glucosurie provoquée par les inhibiteurs des SGLT2 (et donc leur potentialité anti-hyperglycémiante) dépend du degré de l'hyperglycémie alors que la capacité de sécrétion des inhibiteurs de la DPP-4 


\begin{tabular}{|c|c|c|c|}
\hline TABLEAU 3 & \multicolumn{2}{|c|}{$\begin{array}{l}\text { Comparaison des caractéristiques } \\
\text { des inhibiteurs de la DPP-4 } \\
\text { et des inhibiteurs des SGLT2 }\end{array}$} & \\
\hline \multicolumn{4}{|c|}{ HbA1c: hémoglobine glyquée; DFG: débit de filtration glomérulaire. } \\
\hline Propriétés/effets & \begin{tabular}{|l|}
$\begin{array}{l}\text { Inhibiteurs de la DPP-4 } \\
\text { (gliptines) }\end{array}$ \\
\end{tabular} & \multicolumn{2}{|c|}{$\begin{array}{l}\text { Inhibiteurs des SGLT2 } \\
\text { (gliflozines) }\end{array}$} \\
\hline Organe cible & Pancréas endocrine & \multicolumn{2}{|l|}{ Rein } \\
\hline Mécanisme & \begin{tabular}{|l|} 
Insulino-dépendant \\
(en partie) \\
Incrétinopotentiateur \\
Réduction du glucagon
\end{tabular} & \multicolumn{2}{|c|}{$\begin{array}{l}\text { Insulino-indépendant } \\
\text { Glucosurie forcée } \\
\text { Réduction } \\
\text { de la glucotoxicité } \\
\end{array}$} \\
\hline Administration & $\begin{array}{l}\text { Orale, une fois/jour } \\
\text { (sauf vildagliptine } \\
2 \text { fois/jour) } \\
\end{array}$ & \multicolumn{2}{|c|}{ Orale, une fois/jour } \\
\hline Abaissement de l'HbA1c & $-0,7 \grave{a ̀}-0,8 \%$ & \multicolumn{2}{|c|}{$\begin{array}{l}-0,7 \text { à - 1,0\% } \\
\text { (réduction accentuée } \\
\text { si valeur élevée) }\end{array}$} \\
\hline Risque d’hypoglycémie & $\begin{array}{l}\text { Faible (sauf si insuline } \\
\text { ou sulfamide) }\end{array}$ & \multicolumn{2}{|c|}{$\begin{array}{l}\text { Faible (sauf si insuline } \\
\text { ou sulfamide) }\end{array}$} \\
\hline Poids corporel & Neutralité & \multicolumn{2}{|l|}{ Diminution } \\
\hline Pression artérielle & Neutralité & \multicolumn{2}{|l|}{ Diminution } \\
\hline $\begin{array}{l}\text { Manifestations } \\
\text { indésirables }\end{array}$ & $\begin{array}{l}\text { Quasi nulles } \\
\text { (idem placebo) } \\
\text { Risque de pancréatite } \\
\text { controversé }\end{array}$ & \multicolumn{2}{|c|}{$\begin{array}{l}\text { Infections mycosiques } \\
\text { génitales } \\
\text { Infections urinaires } \\
\text { (rares) } \\
\text { Déshydratation/hypo- } \\
\text { tension }\end{array}$} \\
\hline $\begin{array}{l}\text { Utilisation si insuffisance } \\
\text { rénale }\end{array}$ & $\begin{array}{l}\text { Oui (diminution dose } \\
\text { sauf linagliptine) }\end{array}$ & \multicolumn{2}{|l|}{$\begin{array}{l}\text { Non si DFG < } 60 \\
\mathrm{ml} / \mathrm{min} / 1,73 \mathrm{~m}^{2}\end{array}$} \\
\hline $\begin{array}{l}\text { Sécurité cardiovasculaire } \\
\text { démontrée }\end{array}$ & $\begin{array}{l}\text { Oui (SAVOR TIMI 53, } \\
\text { EXAMINE, TECOS) }\end{array}$ & \multicolumn{2}{|c|}{$\begin{array}{l}\text { Oui, avec réduction } \\
\text { mortalité (EMPA-REG } \\
\text { OUTCOME) }\end{array}$} \\
\hline $\begin{array}{l}\text { Utilisation si insuffisance } \\
\text { cardiaque }\end{array}$ & $\begin{array}{l}\text { Oui (pour sitagliptine, } \\
\text { non pour saxagliptine) }\end{array}$ & \multicolumn{2}{|c|}{$\begin{array}{l}\text { Oui (réduction } \\
\text { des hospitalisations) }\end{array}$} \\
\hline Coût journalier & Elevé (versus sulfamide) & \multicolumn{2}{|c|}{ Elevé (versus sulfamide) } \\
\hline
\end{tabular}

s'amenuise en cas d'hyperglycémie sévère, indicatrice d'un épuisement relatif des cellules bêta pancréatiques.

La glucosurie entraîne une perte calorique qui favorise une perte pondérale intéressante, même si celle-ci est en partie freinée par une augmentation compensatoire des ingesta alimentaires. ${ }^{25,27}$ Chez le patient DT2 ou obèse, la perte de poids est un avantage par rapport aux gliptines qui s'avèrent neutres sur le plan pondéral. ${ }^{15}$ Par ailleurs, la glucosurie s'accompagne d'une natriurèse et cet effet diurétique contribue à abaisser la pression artérielle, notamment dans sa composante systolique. ${ }^{28}$ Rappelons que les gliptines ne modifient guère la pression artérielle, ce qui peut représenter un avantage pour les gliflozines chez un patient DT2 hypertendu. Un autre effet des inhibiteurs SGLT2, également expliqué par le mécanisme d'action rénal, est une augmentation de l'élimination urinaire d'acide urique qui conduit à une diminution significative de l'uricémie, un facteur de risque indépendant sur le plan cardiovasculaire et rénal. ${ }^{29}$

Un avantage potentiel des gliflozines est qu'elles peuvent être utilisées avec succès en combinaison avec un agoniste des récepteurs du glucagon-like peptide-1 (GLP-1), ${ }^{30}$ une autre classe ayant démontré une protection cardiovasculaire et rénale dans l'étude LEADER ${ }^{23}$ Ce n'est pas le cas des gliptines qui partagent avec les agonistes des récepteurs du GLP-1 un même mécanisme d'action, ${ }^{31,32}$ et n'offrent donc aucun avantage en combinaison avec ces derniers. ${ }^{33}$
Un avantage significatif en faveur des inhibiteurs des SGLT2 a été apporté par les résultats de l'essai clinique prospectif EMPA-REG OUTCOME qui a démontré une réduction significative des événements cardiovasculaires majeurs et de la mortalité $^{22}$ ainsi que des événements rénaux ${ }^{34}$ chez les patients DT2 avec antécédents cardiovasculaires (donc en prévention secondaire) traités par l'empagliflozine par rapport aux patients recevant le placebo. ${ }^{23}$ Il s'agit là d'un avantage incontestable de l'inhibiteur des SGLT2 par rapport aux inhibiteurs de la DPP-4 chez les patients DT2 à très haut risque cardiovasculaire puisque les gliptines, dans des conditions d'étude et dans des populations DT2 assez comparables, n'ont pu montrer qu'une non-infériorité par comparaison au placebo. ${ }^{21}$ Aussi, l'empagliflozine est désormais reconnue comme un traitement à privilégier chez les patients DT2 avec antécédents cardiovasculaires dans plusieurs recommandations nationales européennes récentes et dans le dernier document résumant le «Standard of medical care» de l'American Diabetes Association. $^{3}$

L'avantage en faveur des inhibiteurs des SGLT2 est, sans doute, le plus important chez le patient DT2 qui présenterait une insuffisance cardiaque ou qui serait à haut risque de développer semblable complication. En effet, on ne peut négliger les résultats contrastés obtenus avec les gliptines (risque accru d'hospitalisation pour insuffisance cardiaque avec la saxagliptine dans SAVOR-TIMI 53 et neutralité avec la sitagliptine dans TECOS) ${ }^{21}$ et avec l'empagliflozine (risque d'hospitalisation pour insuffisance cardiaque réduit significativement de $35 \%$ dans EMPA-REG OUTCOME)..$^{22,23,35}$ L'empagliflozine a d'ailleurs été reconnue comme un traitement d'appoint de l'insuffisance cardiaque dans les dernières recommandations de l'European Society of Cardiology. ${ }^{36}$

Le désavantage des gliflozines par rapport aux gliptines réside dans un profil de tolérance un peu moins favorable, avec, notamment, un risque accru d'infections mycosiques génitales (plus marqué chez les femmes que chez les hommes); les autres effets indésirables sont plus rares, dont les infections urinaires, les hypotensions et les phénomènes de déshydratation, à redouter surtout chez les patients âgés, fragilisés ou sous diurétiques, de l'anse notamment. ${ }^{26}$

Un autre désavantage des inhibiteurs des SGLT2 concerne leur perte d'efficacité en présence d'une insuffisance rénale et ce, en raison même de leur mécanisme d'action rénale spécifique. ${ }^{37}$ Dès lors, cette classe pharmacologique ne représente plus une option thérapeutique validée lorsque le DFG descend en dessous de $60 \mathrm{ml} / \mathrm{min} / 1,73 \mathrm{~m}^{2}$ et, a fortiori, en dessous de $45 \mathrm{ml} / \mathrm{min} / 1,73 \mathrm{~m}^{2}$. Rappelons, comme déjà mentionné précédemment, que la metformine peut être utilisée chez les patients DT2 avec un DFG entre 30 et $60 \mathrm{ml} / \mathrm{min} / 1,73 \mathrm{~m}^{2}$, tout comme les gliptines, moyennant certaines précautions élémentaires, notamment un ajustement de la posologie. ${ }^{7}$ Il faut cependant noter que, dans l'étude EMPA-REG OUTCOME, près d'un quart des patients avaient un DFG compris entre 30 et $60 \mathrm{ml} / \mathrm{min} / 1,73 \mathrm{~m}^{2}$ et que ces patients ont néanmoins bénéficié des avantages de l'inhibiteur des SGLT2 sans manifestation indésirable particulière..$^{34}$ De plus, l'empagliflozine a réduit significativement la survenue d'événements rénaux et, après une réduction initiale transitoire du $\mathrm{DFG}$, a ralenti la chute de ce dernier par rapport au placebo, sans 
doute par un effet intrarénal spécifique (rétrocontrôle tubuloglomérulaire).$^{34}$

Enfin, l'utilisation des inhibiteurs des SGLT2 a été associée à un risque accru d'épisodes d'acidocétose, mais survenus le plus souvent dans des utilisations hors indication (diabétiques de type 1) ou dans des circonstances exceptionnelles (en période post-chirurgicale immédiate, par exemple). ${ }^{38}$ Par ailleurs, il faut noter que la Food and Drug Administration américaine a émis des mises en garde contre un risque possible de fractures et d'amputations des orteils, suite au rapport de quelques rares manifestations indésirables de ce type dans l'étude CANVAS avec la canagliflozine. Un risque accru pour ce type d'événements, non rapportés avec l'empagliflozine et la dapagliflozine, demande cependant confirmation.

Les arguments en faveur et en défaveur de la prescription d'un inhibiteur des SGLT2 après échec d'une monothérapie par metformine sont résumés dans le tableau 2.

\section{ARGUMENTS EN FAVEUR D'UNE ÉVENTUELLE COMBINAISON GLIPTINE-GLIFLOZINE}

Il existe un rationnel pour la combinaison d'un inhibiteur de la DPP-4 et d'un inhibiteur des SGLT2, ${ }^{39}$ ainsi que nous l'avons discuté dans un article précédent de la Revue Médicale Suisse. ${ }^{40}$ La combinaison des deux s'est révélée efficace et bien tolérée. ${ }^{41}$

Ces deux classes pharmacologiques agissent par des mécanismes d'action différents et potentiellement complémentaires. Les inhibiteurs de la DPP-4 ciblent prioritairement le pancréas endocrine (stimulation de la sécrétion d'insuline et diminution de la sécrétion de glucagon, les deux de façon gluco-dépendante). Par contre, les inhibiteurs des SGLT2 ciblent spécifiquement le rein (augmentation de la glucosurie) avec, suite à la réduction de la glucotoxicité, des effets indirects positifs sur la sécrétion d'insuline et la sensibilité à l'insuline, comme déjà mentionné. ${ }^{25}$ Dès lors, il existe un rationnel pour une combinaison de ces deux classes pharmacologiques. ${ }^{39,40}$ A titre d'exemple, les inhibiteurs des SGLT2 augmentent la production de glucagon tandis que les inhibiteurs de la DPP-4 la réduisent, de telle sorte que l'addition d'une gliptine à une gliflozine permettrait de réduire l'augmentation de la production hépatique du glucose rapportée avec les inhibiteurs des SGLT2. ${ }^{25}$ Une réduction plus marquée du taux d'HbA1c a été rapportée avec les combinaisons saxagliptine/dapagliflozine et linagliptine/empagliflozine par comparaison avec chaque antidiabétique pris individuellement, sans aggraver le profil de tolérance, ainsi que nous l'avons rapporté précédemment. ${ }^{40}$

La meilleure preuve que la combinaison des deux approches pharmacologiques présente un intérêt est le fait que deux combinaisons fixes viennent de recevoir l'autorisation de mise sur le marché de l'Agence Européenne du Médicament (EMA), les combinaisons saxagliptine-dapagliflozine ${ }^{42,43}$ et linagliptine-empagliflozine $e^{44,45}$ (tableau 1). Une autre combinaison sitagliptine-ertugliflozine est actuellement en cours d'investigation clinique. Cependant, ce type de combinaison associe deux médicaments plus onéreux, limitation éventuelle dont il conviendra de tenir compte dans une perspective pharmaco-économique.

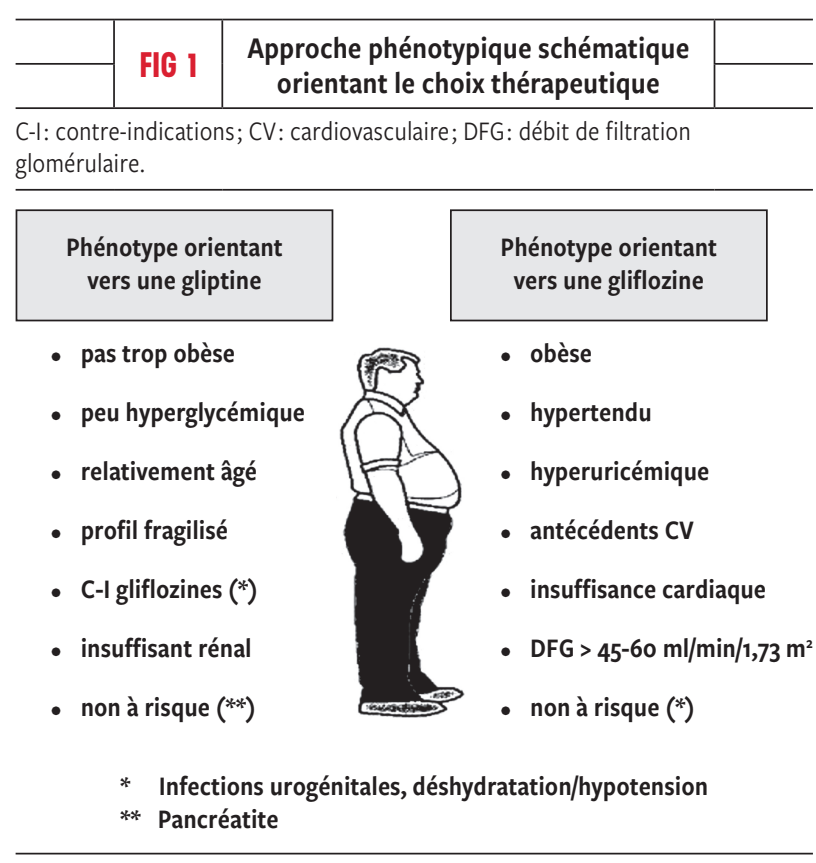

\section{CONCLUSION}

Après échec d'une monothérapie par metformine, le clinicien a le choix entre diverses options thérapeutiques. Parmi les médicaments oraux, deux classes ont pris une place de plus en plus importante, les inhibiteurs de la DPP-4 et des SGLT2. Après échec d'une monothérapie par metformine, il existe des arguments en faveur de l'une ou l'autre classe pharmacologique et le choix peut être orienté selon les caractéristiques individuelles du patient, dans une approche personnalisée (figure 1).

Conflit d'intérêts: N. Paquot a reçu des honoraires comme orateur ou membre d'un conseil scientifique de la part des firmes Merck Sharp \& Dohme, Boehringer Ingelheim et NovoNordisk.

A. Scheen déclare avoir reçu des honoraires comme orateur, membre d'un conseil scientifique ou investigateur clinicien de la part des firmes AstraZeneca, Boehringer Ingelheim, Janssen, Lilly, Merck Sharp \& Dohme, Novartis, NovoNordisk, et Sanofi. Il a été investigateur clinicien dans les études EMPA-REG OUTCOME, TECOS, CANVAS-R et LEADER.

Les données concernant la protection cardiovasculaire et rénale prodiguée par les inhibiteurs des SGLT2, démontrée dans EMPA-REG OUTCOME avec l'empagliflozine, ont été confirmées récemment dans CANVAS avec la canagliflozine. Le lecteur intéressé est invité à prendre connaissance de l'article, publié dans le même numéro, intitulé : Protection cardio-rénale par les inhibiteurs des SGLT2 (gliflozines): d'EMPA-REG OUTCOME à CANVAS. Rev Med Suisse 2017; 13:1421-6. 


\section{MPLICATIONS PRATIQUES}

- Les inhibiteurs de la DPP-4 (gliptines) et les inhibiteurs des SGLT2 (gliflozines) offrent des avantages par rapport aux sulfamides, en termes de risque d'hypoglycémie, de prise de poids et de sécurité cardiovasculaire

- Les gliptines et les gliflozines agissent via des mécanismes d'action différents et chaque classe présente des avantages et des inconvénients, susceptibles de guider le choix du clinicien dans une approche personnalisée

- Après échec d'une monothérapie par metformine, le choix entre l'ajout d'une gliptine ou d'une gliflozine peut être orienté par certaines caractéristiques cliniques et biologiques d'accès aisé pour le clinicien

1 Inzucchi SE, Bergenstal RM, Buse $J B$, et al. Management of hyperglycaemia in type 2 diabetes, 2015: A patient-centred approach. Update to a position statement of the American Diabetes Association and the European Association for the Study of Diabetes. Diabetologia 2015:58:429-42.

2 Garber AJ, Abrahamson MJ, Barzilay $\mathrm{JI}$, et al. Consensus statement by the American Association of Clinical Endocrinologists and American College of Endocrinology on the comprehensive type 2 diabetes management algorithm - 2016 executive summary. Endocr Pract 2016; 22: 84-113. 3 American Diabetes Association. Standards of medical care in diabetes - 2017. Diabetes Care 2017:40:S1-S135. 4 * Scheen AJ, Paquot N. Actualisation 2015 du traitement de l'hyperglycémie dans le diabète de type 2 . Rev Med Suisse 2015;11:1518-5.

5 Scheen AJ, Lefèbvre PJ. Ora antidiabetic agents. A guide to selection. Drugs 1998:55:225-36. 6 UKPDS group. Effect of intensive blood-glucose control with metformin on complications in overweight patients with type 2 diabetes (UKPDS 34). UK Prospective Diabetes Study (UKPDS) Group. Lancet 1998;352:

854-65.

7 Scheen AJ. Utilisation des antidiabétiques oraux en cas d'insuffisance rénale. Rev Med Suisse 2012;8:1614-20. 8 Scheen AJ. A review of gliptins for 2014. Exp Opin Pharmacother 2015;16:43-62

9 Scheen AJ. Pharmacodynamics, efficacy and safety of sodium-glucose co-transporter type 2 (SGLT2) inhibitors for the treatment of type 2 diabetes mellitus. Drugs 2015;75:33-59. 10 Guthrie RM. Clinical use of dipeptidyl peptidase- 4 and sodium-glucose cotransporter 2 inhibitors in combination therapy for type 2 diabetes mellitus. Postgrad Med 2015;127:463-79.
11 Scheen AJ. SGLT2 versus DPP4 inhibitors for type 2 diabetes. Lancet Diabetes Endocrinol 2013;1:168-70. 12 *Scheen AJ, Paquot N. Le choix entre un inhibiteur de la DPP-4 et un inhibiteur des SGLT2 pour traiter le diabète de type 2 . Rev Med Liege 2016;71:579-85.

13 Scheen AJ. Comment je traite ... Le choix entre un sulfamide hypoglycémiant et une gliptine pour traiter

le diabète de type 2 . Rev Med Liege 2014;69:476-84.

14 Scheen AJ. Comment je traite ... A propos du positionnement des inhibiteurs de la DPP-4 (gliptines) dans le traitement du diabete de type 2 . Rev Med Liege 2015;70:593-9.

15 Van Gaal L, Scheen A. Weight management in type 2 diabetes: Current and emerging approaches to treatment. Diabetes Care

2015;38:1161-72.

16 Scheen AJ. Dipeptidylpeptidase-4 inhibitors (gliptins): Focus on

drug-drug interactions. Clin Pharmacokinet 2010;49:573-88.

17 Scheen AJ. Safety of dipeptidy peptidase-4 inhibitors for treating type 2 diabetes. Expert Opin Drug Saf 2015;14:505-24.

18 Paolisso G, Monami M, Marfella R, et al. Dipeptidyl peptidase-4 inhibitors in the elderly: More benefits or risks? Adv Ther 2012;29:218-33.

19 Scheen AJ. Pharmacokinetics and clinical use of incretin-based therapies in patients with chronic kidney disease and type 2 diabetes. Clin Pharmacokinet 2015;54:1-21. 20 Abdelmoneim AS, Eurich DT, Light $\mathrm{PE}$, et al. Cardiovascular safety of sulphonylureas: over 40 years of continuous controversy without an answer. Diabetes Obesity Metab 2015;17:523-32.

21 Scheen AJ. Gliptines, sécurité cardiovasculaire et insuffisance cardiaque: le point après l'étude TECOS. Rev Med Suisse 2015;11:
1518-25.

22 Zinman B, Wanner C, Lachin JM et al. Empagliflozin, cardiovascular outcomes, and mortality in type 2 diabetes. N Engl J Med 2015;373: 2117-28.

23 Scheen AJ, Wallemacq C, Jandrain $B$, et al. Protection cardiovasculaire du patient diabétique de type 2: d'EMPAREG OUTCOME à LEADER. Rev Med Suisse 2016;12:1370-5.

24 Abdul-Ghani MA, Norton L, DeFronzo RA. Role of sodium-glucose cotransporter 2 (SGLT 2) inhibitors in the treatment of type 2 diabetes. Endocr Rev 2011;32:515-31. 25 Scheen AJ, Paquot N. Metabolic effects of SGLT2 inhibitors beyond increased glucosuria: A review of clinical evidence. Diabetes Metab 2014;40:S4-S11.

26 Scheen AJ. SGLT2 inhibitors: Benefit/risk balance. Curr Diabetes Rep 2016;16:92

27 Ferrannini G, Hach T, Crowe S, et al. Energy balance after sodium glucose cotransporter 2 (SGLT2) inhibition. Diabetes Care 2015;38:1730-5. 28 Scheen AJ. Effects of reducing blood pressure on cardiovascular outcomes and mortality in patients with type 2 diabetes: Focus on SGLT2 inhibitors and EMPA-REG OUTCOME. Diabetes Res Clin Pract 2016;121:204-14.

29 Schils R, Krzesinski JM. Hyperuricémie et risque potentiel de pathologie cardio-vasculaire et rénale. Rev Med Liege 2016;71:262-8.

30 Frias JP, Guja C, Hardy E, et al. Exenatide once weekly plus dapagliflozin once daily versus exenatide or dapagliflozin alone in patients with type 2 diabetes inadequately controlled with metformin monotherapy (DURATION-8): A 28 week, multicentre, double-blind, phase 3 , randomised controlled trial. Lancet Diabetes Endocrinol 2016;4:1004-16. 31 Scheen AJ. GLP-1 receptor agonists or DPP-4 inhibitors: How to guide the clinician? Ann Endocrinol (Paris) 2013;74:515-22.

32 Nauck M. Incretin therapies: Highlighting common features and differences in the modes of action of glucagon-like peptide- 1 receptor agonists and dipeptidyl peptidase-4 inhibitors. Diabetes Obesity Metab 2016;18:203-16

33 Nauck MA, Kahle M, Baranov O, et al. Addition of a dipeptidyl peptidase- 4 inhibitor, sitagliptin, to ongoing therapy with the glucagon-like peptide-1 receptor agonist liraglutide: A randomized controlled trial in patients with type 2 diabetes. Diabetes Obesity Metab 2017;19:200-7.

34 Wanner C, Inzucchi SE, Lachin JM et al. Empagliflozin and progression of kidney disease in type 2 diabetes.
N Engl J Med 2016;375:323-34.

35 Fitchett D, Zinman B, Wanner C, et al. Heart failure outcomes with empagliflozin in patients with type 2 diabetes at high cardiovascular risk: Results of the EMPA-REG OUTCOME ${ }^{\circledR}$ trial. Eur Heart J 2016;37:1526-34. 36 Ponikowski P, Voors AA Anker SD, et al. 2016 ESC Guidelines for the diagnosis and treatment of acute and chronic heart failure: The Task Force for the diagnosis and treatment of acute and chronic heart failure of the European Society of Cardiology (ESC). Developed with the special contribution of the Heart Failure Association (HFA) of the ESC. Eur J Heart Fail 2016;18:891-975.

37 Scheen AJ. Pharmacokinetics, pharmacodynamics and clinical use of SGLT2 inhibitors in patients with type 2 diabetes and chronic kidney disease. Clin Pharmacokinet 2015;54:691-708.

38 Rosenstock J, Ferrannini E. Euglycemic diabetic ketoacidosis: A predictable, detectable, and preven table safety concern with SGLT2 inhibitors. Diabetes Care

2015;38:1638-42.

39 Scheen AJ. DPP-4 inhibitor plus SGLT-2 inhibitor as combination therapy for type 2 diabetes: From rationale to clinical aspects. Expert Opin Drug Metab Toxicol 2016;12:1407-17.

40 *Scheen AJ, Paquot N. Combinaison gliptine-gliflozine dans le traitement du diabète de type 2 . Rev Med Suisse 2016;12:1384-8.

41 Dey J. SGLT2 inhibitor/DPP-4 inhibitor combination therapy - complementary mechanisms of action for management of type 2 diabetes mellitus. Postgrad Med 2017;129:409-20.

42 Scheen AJ. Pharmacokinetic drug evaluation of saxagliptin plus dapagliflozin for the treatment of type 2 diabetes. Expert Opin Drug Metab Toxicol 2017;13:583-92.

43 Garnock-Jones KP. Saxagliptin/dapagliflozin: a review in type 2 diabetes mellitus. Drugs 2017;77:319-30.

44 Triplitt C, Solis-Herrera C, Cersosimo $E$, et al. Empagliflozin and linagliptin combination therapy for treatment of patients with type 2 diabetes mellitus. Expert Opin Pharmacother 2015;16:2819-33.

45 Aronson R. Single-pill combination therapy for type 2 diabetes mellitus: Linagliptin plus empagliflozin.

Cur Med Res Opin 2015;31:901-11.

$$
\begin{aligned}
& \text { * à lire } \\
& \text { ** à lire absolument }
\end{aligned}
$$

